Canadian Journal of Higher Education Revue canadienne d'enseignement supérieur

Volume 44, No. 1, 2014, pages 22 - 37

\title{
Internet research ethics and the policy gap for ethical practice in online research settings
}

Jacqueline G. Warrell \& Michele Jacobsen

University of Calgary

\begin{abstract}
A growing number of education and social science researchers design and conduct online research. In this review, the Internet Research Ethics (IRE) policy gap in Canada is identified along with the range of stakeholders and groups that either have a role or have attempted to play a role in forming better ethics policy. Ethical issues that current policy and guidelines fail to address are interrogated and discussed. Complexities around applying the human subject model to internet research are explored, such as issues of privacy, anonymity, and informed consent. The authors call for immediate action on the Canadian ethics policy gap and urge the research community to consider the situational, contextual, and temporal aspects of IRE in the development of flexible and responsive policies that address the complexity and diversity of internet research spaces.
\end{abstract}

\section{RÉSUMÉ}

Un nombre croissant de recherchistes en enseignement et en sciences sociales conçoivent et dirigent des recherches en ligne. La présente revue identifie les lacunes en matière de politique d'éthique en recherche Internet (Internet Research Ethics - IRE) au Canada, et reconnaît l'éventail d'intervenants et de groupes qui ont soit joué un rôle, soit tenté d'en jouer un, dans la création d'une meilleure politique d'éthique. On y aborde les enjeux éthiques 
auxquels les politiques et lignes directrices actuelles ne répondent pas et on s'interroge à ce sujet. On y explore les complexités relatives à l'application du modèle humain à la recherche dans Internet, comme les enjeux portant sur l'anonymat, le consentement éclairé et le respect de la vie privée.

Les auteurs invitent à passer immédiatement à l'action en ce qui a trait aux lacunes en matière de politique d'éthique au Canada, et pressent le milieu de la recherche afin qu'il prenne en considération les aspects situationnels, contextuels et temporels de l'éthique en recherche Internet dans la création de politiques souples et judicieuses qui abordent la complexité et la diversité des espaces de recherche Internet.

\section{Introduction}

Current growth in social networking, online digital media production and consumption, and cloud and mobile computing makes the internet an exciting and engaging environment for formal and informal learning and playful interactions (Jacobsen, 2010; Thomas \& Brown, 2011). Digital and social technologies tend to change how people of all ages learn, collaborate, play, socialize, access resources and services, and connect (Jacobsen \& Friesen, 2011). Ongoing research is needed to better understand how and why people play and participate in online communities and join global collectives to work collaboratively to design, develop, implement, assess, and discuss their strategies, solutions, goals, and ideas and to build upon other's digital expressions and creations. Educational researchers both require and have designed new online research methods to study the use of the internet and digital media for connecting, communicating, teaching, and learning in both formal and informal environments.

Research is expanding from conventional and well-developed methods of on-site, inperson, real-time data collection, such as face-to-face interviews, telephone surveys, and mail-out questionnaires, to include virtual observation, online surveys, and data collection in spaces such as blogs, websites, chat rooms, social media sites, and so on. The shift towards more online and blended educational, social, and recreational engagements, along with the use of evolving research methods to study online behaviours and interactions, creates new questions about the ethics of online data collection. As a research community, we need to consider how educational research will be designed and conducted ethically in online settings. In this paper, we argue that a current Internet Research Ethics (IRE) policy gap exists in Canada, and we identify the range of stakeholders and groups that either have a role or have attempted to play a role in forming policy. We interrogate some of the ethical issues that current policy and guidelines fail to address. Privacy, informed consent, anonymity, and confidentiality have been, at best, only partially attended to in ethical policies and guidelines, which are insufficient for guiding internet researchers on ethical decision making. In our conclusion, we call for immediate attention to the IRE policy gaps in the Canadian Tri-Council Policy Statement (2010), and urge the research community to consider the situational, contextual, and temporal aspects of IRE in the development of flexible and responsive policies that address the complexity and diversity of internet research spaces. 


\section{Internet Research Ethics and the Canadian Policy Gap}

Internet Research Ethics is a topic that has been of growing interest to researchers since the early nineties as internet use and broadband subscriptions have steadily increased. A problem researchers face is that while research interest and scholarly activity in online behaviour and interactions has grown, and new research methods and approaches to data collection have been developed, the ethical guidelines and policy to inform research in online contexts is still in its infancy.

In 1996, The Information Society published a special edition on IRE. King's (1996) contribution to this publication is still widely cited in the field. In 1999, Frankel and Siang published an IRE report for the American Association for the Advancement of Science (AAAS) and in 2002 the Association of Internet Researchers (AoIR) ethics working group developed their own set of guidelines. Since that time, a number of works have been published by researchers across disciplines on the topic of IRE (Beaulieu \& Estalella, 2011; Buchanan \& Ess, 2009; Buchanan \& Ess, 2008; Estalella \& Ardevol, 2007; Kitchin, 2007; McKee \& Porter, 2009).

In Canada, in spite of growing interest in carrying out research in online settings and using online methods (Kitchin, 2007), we contend that the policy to guide these practices is insufficient. The Canadian Tri-Council Policy Statement (2010; TCPS2), developed by the Interagency Advisory Panel on Research Ethics, the Canadian Institutes of Health Research (CIHR), the Natural Sciences and Engineering Research Council of Canada (NSERC), and the Social Sciences and Humanities Research Council of Canada (SSHRC), has established common standards for ethical research practices in Canada. The TCPS2 makes very limited reference to internet research in only three sections of the document. IRE is mentioned with regard to the requirement for an ethics review, with regard to the security of collected data, and very briefly, regarding observational studies. The three directives amount to little or no guidance for navigating diverse issues of internet research, such as recruitment, obtaining informed consent in dynamic communities and from static online archives, the continuum of public versus private spaces, and estimating risk.

Similar to the initial, 1998 version of the national ethics policy, the most recent TCPS2 (2010) fails to adequately address IRE. The policy gap has led to a lack of consensus concerning the ethical practice of research on the internet (Kitchin, 2007). A similar situation exists in the United States, where the federal regulations for human subject research, Title 45, Part 46 of the Code for Federal Regulations also fails to address IRE (McKee \& Porter, 2009). Based on a review of literature and interviews with internet researchers in the United States, McKee and Porter (2009) concluded, "this lack of guidance at the federal level impacts IRBs [Institutional Review Boards, the Canadian equivalent of Research Ethics Boards] at the local level" (p. 35). Ethics review processes for social science research in the US can be rife with misconceptions, inconsistencies in decision making, and mission creep in the oversight by IRBs (Schrag, 2011). Given the policy gap on IRE, individual Canadian Research Ethics Boards (REBs) who refer to the current Tri-Council guidelines are likely to develop their own internal, often opaque, decisionmaking practices (Schrag, 2011), which can diverge greatly from institution to institution (Charbonneau, 2005) and tend to cause significant problems and delays for researchers who submit research protocols that involve emerging forms of online research. Similar 
to Charbonneau's (2005) experience of receiving different advice and requirements to revise a survey protocol from each of four ethics review boards at four different Canadian universities, the second author of this article received different advice and diverse requirements to revise in response to an online research protocol from two university REBs. One REB approved the protocol and requested only minor revisions, and the second REB requested substantive revisions to recruitment methods, consent protocols, and online survey methods, which delayed the start of the research. Without clear guidance from the current set of ethical guidelines and faced with different internal decision-making practices by REBs, researchers have to rely on the research literature, popular practices, and their own collegial networks to design ethics protocols for online research settings and contexts. With individual REBs likely to develop homegrown practices to fill the policy gap and with a lack of clear policy at the national level, researchers are in the disadvantaged position of trying to hit a moving target in the preparation of ethics proposals to conduct internet research.

\section{Key Issues and Complexities of Internet Research Ethics}

The TCPS2 is developed specifically for research involving human subjects (CIHR et al., 2010a). This is a particularly pertinent point for researchers who may attempt to adapt the TCPS2 to the online setting. In subsequent sections of this paper, the wideranging debate over the question of whether all internet research should be considered human subjects' research or not is explored. Several key issues, such as types of internet research, private versus public spaces, obtaining informed consent online, and anonymity, for which REBs and researchers need clear and current guidance, are discussed.

\section{Is Internet Research Human Subject Research or Not?}

Research is conducted over the internet using a variety of methods. Kitchin (2007) divided internet research into two main categories, web-based research and online research. Web-based research is further sub-divided into non-intrusive and engaged research. Nonintrusive web-based research refers to the collection and analysis of existing data on the web. This type of research involves no interaction with participants and the researcher, as observer, has no influence on the creation of data (i.e., no intervention). In contrast, during engaged web-based research, the researcher, still acting as observer, interacts with the participants, for example, to solicit feedback on their analysis of the data to ensure understanding and/or to seek clarification. Online research refers to situations where the researcher places him or herself within the research context, becoming an active participant-observer in the online setting. Data collection involves significant interaction between the researcher and the participants. Examples of online research can include participatory methods or ethnography, among other educational research methods.

The distinction between the different types of internet research is important when considering ethical issues and practices. Each type of research involves different levels of involvement and interaction on the parts of both the participant and the researcher. The more involvement and interaction, the greater, one can assume, the ethical risk may be (Kitchin, 2007). Additionally, only research determined to be human subject research is subject to review by REBs (CIHR et al., 2010a). 
Walther's (2002) definition of human subject research has two key components. First, human subject research requires interaction between the researcher and the participant. Second, the data collected is potentially traceable, either directly or indirectly, to individual participants. The TCPS2 differentiates human subject research from other research as,

research that is non-intrusive, and does not involve direct interaction between the researcher and individuals through the Internet... Cyber-material such as documents, records, performances, online archival materials or published third party interviews to which the public is given uncontrolled access on the Internet for which there is no expectation of privacy is considered to be publicly available information. (CIHR et al., 2010a, p. 18)

Non-intrusive web-based research is usually not considered human subject research and therefore is not subject to an ethics review. However, review boards that are misinformed or populated by reviewers who lack sufficient disciplinary knowledge about internet research may too hastily exempt research projects from the ethics review process (McKee \& Porter, 2009). McKee and Porter (2009) described an example of one research project that examined the personal webpages created by teenage girls. The researcher had no interaction with the creators of the websites. Therefore, the research was determined not to involve human subjects and an ethics review was not required. At one point during the study, one girl posted information on her webpage indicating she may inflict harm on herself. The researcher, under the impression this was not human subject research, did not respond the girl's assertion and failed to take any further action or follow-up. This is only one example that highlights the complex nature of internet research and suggests current definitions of human subject research may not translate well to online research contexts.

The last line in the definition of human subject research from the current TCPS2, "no expectation of privacy," is key and alludes to an ongoing debate among scholars who question if information posted on the internet should be considered public or private, and what role, if any, that REBs should play in overseeing such research.

\section{Public Versus Private Space}

The ongoing debate as to whether the internet should be considered a private or a public space has significant implications for IRE. As outlined in the TCPS2, non-intrusive, minimal risk research conducted in public spaces is not subject to the same ethical considerations or to the ethics review process as research conducted in private spaces (CIHR et al., 2010a). The diversity of opinions on this matter is great and most commentators define levels of privacy using a continuum. Some internet sites viewed as more private spaces require a person to register as a member using their personal information. Even more restrictive sites may require the individual to pay a registration fee or be approved by a moderator before joining the online community. Others are completely restriction free, open for anyone to view and post information. Kitchin (2007) and others advocated that research, using restriction-free sites may be compared to research conducted in "offline" public spaces, such as a park or a shopping mall. Kitchin (2007) took a strong stance on these non-restricted online spaces and asserts, "it is unreasonable to assume pre-existing cybertext to be private in nature" (p. 64). She likened the assignment of "privacy" of existing cybertext to a "community member in a town square and speaking loudly 
enough for people around to hear, but expecting the observers to not pay attention" (p. 64). Moreno, Frost, and Christakis (2008) provided a different analogy, comparing nonintrusive web-based research to eavesdropping on a conversation in a coffee shop, where someone close by may listen in, but there is little risk of being recorded. These comparisons raise two important issues: (1) intended audience, and (2) perceived privacy.

Intended audience. In both the town hall and coffee shop examples, although the conversations are "held in a public place, [they] are intended for a private audience" (Moreno Frost, and Christakis, 2008, p. 158). The speaker chose to make statements based on the people physically present at that time, and knowing that those who are present but who are not explicitly a part of the intended audience, may still listen in. This differs from an online conversation, where it can be difficult for an individual to verify who is reading or has access to the statements they post. Research shows that people who make online statements on websites and in discussion forums are often under the impression that a certain audience is reading their posts. In a study by Elgesem (2002), it became obvious to the researcher that the online participants in a discussion forum for lesbians assumed that they were posting to a specific audience, a community of other lesbians and those supportive of their lifestyle. Elgesem (2002) also noted how this presumed audience led to the perception of privacy and the sharing of highly personal information often of a sensitive nature.

Perceived privacy. One could argue that expectations and awareness of privacy issues have changed over the past 10 years as more people take their conversations online and join various online communities. Elgesem (2002) concluded that an important difference exists between offline and online settings regarding sharing and accessibility of information. In offline settings, he suggests that accessibility and sharing of sensitive information are inversely related. The more restricted the audience (the less people that have access), the more likely someone is to share sensitive information. Privacy is therefore related to the audience, which is linked to the person's level of disclosure. However, this does not hold true in the online world where perceived privacy and trust may lead to sharing sensitive information despite the realistic potential of that information being widely accessible to others (Elgesem, 2002). Participants in online spaces often develop expectations of privacy and confidentiality that are unrealistic and do not reflect the actual situation. Estalella and Ardevol (2007) argued that it is not the technology itself or the accessibility of websites that determine a space to be public or private. Instead, they refer to Allan's use of "situational ethics" to consider the many aspects that influence a participant's perceived privacy (as cited in Estalella \& Ardevol, 2007, p. 40). Therefore, deciding whether an online space should be considered public or private may require the researcher and REB to make a judgment call. In making this judgment call, researchers may consider some of the following questions:

1. What type of information is being shared? Is it simple facts and everyday conversations, or are the topics more personal and intimate? Do discussions include people's closely held values and beliefs?

2. How accessible is the online space? Is it open for anyone to join, and are newcomers or comments by anyone welcomed? A greater level of perceived privacy is associated with online communities that have registration requirements (Eysenbach \& Till, 2001; King, 1996). 
3. Can users select privacy settings and control who can access their online information? For example, Facebook allows users to control who can view their information. Facebook users can chose to make their information viewable by everyone, only by accepted friends within their network, or by friends of their friends, or to no one. A person who chooses to make their information public to everyone likely has a lower level of perceived privacy than someone who sets their privacy restrictions higher (Ess et al., 2002).

4. Who are the participants in an online group and what are the group's norms? A group with a thousand-plus members may be thought to be more of a public space than a group with fifteen members who share personal stories, develop a sense of trust, and seem to expect confidentiality (Eysenbach \& Till, 2001).

5. What is the purpose or aim of the speaker, the conversation, or the online forum, and who is the intended or assumed audience? Is the purpose to make a political statement or to broadcast and disseminate information? Could participants experience negative consequences if their discussions and statements were shared outside of the group or intended audience, and who is responsible for this risk? Researchers should also bear in mind that they are not likely a part of the assumed audience and that their presence may be viewed as an intrusion (Moreno, et al., 2008).

This distinction between public and private online spaces is not clear-cut and it has significant implication for IRE, specifically for non-intrusive web-based research. If nonrestrictive online spaces are deemed to be public spaces and thereby also falling outside of human subject research, any social science researcher should be able to carry out this type of internet research without having to apply for ethics review. Researchers may also carry out such research without informed consent. The TCPS2 is largely silent on whether ethics review is required for research in public online spaces.

\section{Informed Consent}

Ethical decisions involving informed consent should consider questions such as, when is informed consent required, how can it be obtained, and how can it be validated?" (Frankel \& Siang, 1999, p. 7). It can be a challenge to confirm the identity of participants in online settings, and it can be a challenge to get informed consent for existing information. For example, people might use an avatar or adopt a username to participate in an online discussion or service, and it may be difficult or impossible to contact the person who owns that avatar or username. For existing blogs, Twitter feeds, discussion groups, or online communities, there may be contributions from weeks or years before from users or participants who have long since moved on, or it may no longer be an active account. It may be impossible to contact these individuals for consent to analyze their contributions. We contend that available online content should not automatically be excluded from research attention and analysis because of the requirement to obtain informed consent.

When should informed consent be required? If parts of the internet are viewed as a public domain, the TCPS2 does not mandate researchers using non-intrusive webbased research to obtain informed consent. From this point of view, information posted on the web is posted in a public space, easily accessed by anyone, including researchers, and is therefore open to being included in research data (Kitchin, 2007). Some studies have suggested that identifiable research participants do not share this same view and 
have strong opposing feelings (Estalella \& Ardevol, 2007; Eysenbach \& Till, 2001; King, 1996). In a study by King (1996), participants from an online support group responded negatively to being quoted without their permission, despite their postings being publically accessible. Estalella and Ardevol (2007) also found that participant's comments suggested that use of their blogs for research purposes without consent was an invasion of privacy and had negative consequences for the online group. Failure to seek consent or to disclose oneself as a researcher participating in an identifiable group risks violating a group's perceived privacy and their sense of trust and security (King, 1996). In some cases, the group may fail to re-establish this trust and the supportive environment that once allowed for rich, deep, and personal conversations is never restored (Estalella \& Ardevol, 2007).

However, choosing not to seek consent also has advantages. Primarily, the online community and its members are not influenced by the presence of the researcher (Estalella \& Ardevol, 2007). Participants may change their behaviour if they know they are subjects of a research study. Because the goal of educational research is to improve teaching and learning, gaining a true understanding and accurate picture of the participants' naturally occurring online behaviour is vital. Some balance needs to be found whereby informed consent is considered in the context of the date, availability, and traceability of the online information.

In comparison to observational research conducted in a physical public space, the ease with which a researcher can unobtrusively "lurk" and invisibly collect data in the online setting may make this a particularly pertinent ethical consideration. Deception is also more easily carried out in the online environment (Frankel \& Siang, 1999). For example, a researcher could easily join an online discussion forum using either real or imaginary personal details and participate in that community without disclosing their identity as a researcher. One of the primary concerns of internet research involving deception is the difficulty in debriefing the participants (Frankel \& Siang, 1999). In real-life research settings, participants are typically debriefed at the end of the study, or if the participant leaves the study, at the time of doing so. Participants on the internet tend to come and go and can be difficult to track. Similarly, online participants may choose to change their screen names making it additionally hard for researchers to remain connected with them (Buchanan \& Ess, 2008; Walther, 2002). Issues of deception, informed consent, and debriefing raise important considerations for educational and other social science researchers who need to weigh the advantages of the greater good with the risks to the individual and their online community when deciding whether to seek consent and/or attempt to debrief participants.

How can informed consent be obtained and validated? There are different ways that informed consent may be obtained through the internet, including email or online statements requiring participants to select an agreement checkbox to express their consent. The AoIR reminds researchers of the temperamental nature of electronic methods, such as those that may fail, become erased, or be corrupted (Ess \& Association of Internet Researchers, 2002). The non-physical presence of participants in the virtual world also makes it difficult for researchers to verify certain information. The TCPS2 requires researchers to verify the age and the mental capacity of individuals if seeking informed consent. Although, verification of age and capacity can also be difficult with conventional 
methods of research (in-person, telephone interviews, or mail-out questionnaires), Walther (2002) highlighted the ease and increased frequency with which one can and does misrepresent themselves on the internet and the additional challenge this may present for researchers who may be unable to verify information such as age. The difficulty of verifying participants' age in online environments may raise additional concerns about obtaining parental consent for internet research with children. The TCPS2 stipulates that informed consent should be sought from an appropriate third party (i.e., parent or guardian) for participants who are children. Questions about when additional consent should be sought and methods for obtaining consent via the internet highlight this as an area requiring further attention and research.

Lastly, Moreno et al. (2008) suggested that researchers review site policies associated with the online setting they aim to research. Different websites, discussion forums, and online communities have different policies in place regarding research and ownership of information. This may restrict what information can be used, how it is used, and how participants may be represented in the written results, including issues of copyright versus anonymity.

\section{Anonymity}

Internet research has sparked an interesting debate about the difference between anonymity and copyright. In Canada, "copyright applies to all original literary, dramatic, musical, and artistic works. These include books, other writings, music, sculptures, paintings, photographs, films, plays, television and radio programs, and computer programs. Copyright also applies to other subject matter, which includes sound recordings (such as records, cassettes, or compact discs), performer's performances, and communication signals (Canadian Intellectual Property Office, 2008). Copyright includes any works on the internet. A great deal of information on the internet is not posted anonymously. The author either identifies themselves by their real name or by an online screen name or nickname. The ethical dilemma for researchers is whether one should or even may give credit to the source of the information/data, and if the author is identifiable, whether or not it is appropriate to preserve the person's anonymity (Buchanan \& Ess, 2008). The AoIR guidelines asked researchers to consider whether they view their participants as human subjects or as authors (Ess \& Association of Internet Researchers, 2002). If viewed more as an author, the researcher may lean towards giving credit where credit is due. If however, the participant is viewed as a human subject to be protected, the researcher is likely to ensure the individual's anonymity is maintained. Anonymous participation is part of the attempt to minimize any harms or risks to reputation, professional standing, or other personal or professional matters that may result from that person being associated with the research or its findings. However, if the participant is considered an author, anonymity deprives the contributor of credit for their work.

The decision to list online participants as anonymous participants or as authors is complex and the lines are blurred; context is key. Bassett and O'Riordan (2002) described their study involving a discussion board for the lesbian community. This discussion board was open and accessible to anyone, however the researchers determined it to be a semiprivate space. This decision was made based on user's confessional posts and suggestions that it was safe place to raise ideas and questions not accepted elsewhere. Overestimating 
risk and taking the safest option available regarding anonymity, the researchers removed from their findings any information that might have identified the discussion forum or its members. In retrospect, Bassett and O'Riordan (2002) questioned their decision, suggesting they may have been too quick to apply the human subject model of research to this situation. The particular online setting that they studied was not just a space for chatting and discussion; it also had an inherent political agenda. It gave lesbians a voice and a channel to make their opinions and beliefs heard. By not disclosing the website or any of the participants, Bassett \& O'Riordan (2002) argued that they may have inadvertently reinforced homophobia, contributed to further silencing the group, and marginalized them. In this case, the researchers argued that what appeared to be the most ethical option at the time could be seen as unethical in hindsight. This example speaks to the power of research to be emancipatory and empowering for participants. These scenarios again call for researchers and research ethics boards to apply "situational and contextual ethics" that attempt to take into consideration the participants' wishes, the sensitivity of the information, and the potential benefits versus the potential harms in an open and transparent manner.

If anonymity is to be maintained, the researcher needs to consider how this will be accomplished. Common practice, according to a number of the guidelines referenced in this paper, is to report only general findings or to use pseudonyms. During internet interactions, people use pseudonyms on a regular basis. Nicknames or screen names are often used in chat rooms, on social networking sites, and on websites. Screen names can be used both to conceal a person's real identity and also to intentionally represent oneself in a particular way. It may be tempting for researchers to use the pseudonym the person has already created or constructed for himself or herself, however Buchanan and Ess (2008) and Frankel and Siang (1999) cautioned against this practice. Screen names are often comparable "to real identities in the online world" (Frankel \& Siang, 1999). Disclosing an individual's online persona may be just as harmful as revealing their true identity.

Unlike offline content, online content is easily searchable by Google and other internet search engines. A Google search of screen names used in a research report could result in the easy identification of individuals. Additional verbatim quotes or the use of key phrases included in the researcher's written findings may lead to participants or sites of research being identified. Therefore, researchers should take into account the accessibility and searchability of nicknames, screen names, and quotes when preparing reports of their research.

Kitchin (2007) suggests that internet research may actually provide greater anonymity to participants whose geographical locations are also usually concealed from researchers and others. However, this may be less true with the emergence and growing popularity of websites like Foursquare and other social networking applications that invite users to post their current geographical location. Therefore, geographical locations are now often tied to screen names and to individual contributions, like a tweet or photograph. Researchers need to be cognizant of the fast pace with which online applications are changing. While it may have been a fair assumption that a participant's location could not be traced by their screen name several years ago, this is less true today. All these issues and ambiguities surrounding IRE highlight the need for more guidance for internet researchers. 


\section{Addressing the Canadian Policy Gap}

The current TCPS2 (2010) guidelines demonstrate some little progress from the last amended version of the TCPS in 2005, which made no reference to IRE at all. Yet, its superficial treatment of online research contexts and issues still highlight the need for more attention to be given to the unique issues that arise in online research.

\section{Diverse Perspectives and Voices on Ethics Policy}

In 2008, in response to the gaps in the 1998 version of TCPS, a SSHRC Special Working Committee (SSHWC) prepared a 10-page report that explored unique considerations for the ethical practice of internet research. Based on the work of Ess and the Association for Internet Researchers (2002), the National Committee for Research Ethics in the Social Sciences and the Humanities (2003)-a Norwegian ethics committee-and Kitchin (2007), the report makes several recommendations. It raises several ethical issues, such as privacy, informed consent, anonymity, and data security. The authors superficially explore the issues, in some cases offering some general recommendations for good practice rather than clear guidance for internet researchers. For example, with regard to informed consent, the report provides some suggestions for how informed consent may be obtained in an online environment but does not clearly identity if and when informed consent should be required. No recommendations are provided regarding privacy, anonymity, and confidentiality; nor does it address the assessment of participant risk associated with internet research. The SSHWC report simply concludes that this issue "remains open" to ethical debate, and unfortunately, it fails to acknowledge the many complexities of ethical decision making in online research. For example, the report's discussion of anonymity makes no reference to the traceability of internet data when disseminating results or the potential harm that may be experienced by participants or online groups who may be exposed. Both are key issues for consideration by internet researchers (Beaulieu \& Estalella, 2011; McKee \& Porter, 2009).

What is more concerning than the SSHWC's superficial exploration of key ethical issues in online research is the absence of any of its recommendations in the most recent version of TCPS2 (CIHR et al. \& Queen's University, 2010ab). Though the committee acknowledges and recommends that "the ethical issues associated with internet-based research are many, and they should be taken into consideration in the new version of the TCPS" (SSHWC, 2008, p. 9), the TCPS2 still fails to address the many ethical issues and recommendations regardings online research in its guidelines. The new TCPS2: CORE Tutorial, developed by the Canadian Institutes of Health Research (CIHR) and Queen's University (2010b), is designed to help researchers and members of REBs to further explore and apply the TCPS2. At some Canadian universities, such as the University of Calgary, graduate students are required to demonstrate that they have completed the TCPS2: CORE Tutorial when they apply for ethical clearance for their research. However, the TCPS2: CORE Tutorial, like other guidelines and materials from the Tri-Council, makes few and only general references to online research; not one of the scenarios that give researchers an opportunity to practise applying ethical decision making incorporates the internet or any form of online community or interaction as the research context and environment as an example. 
Although the Interagency Advisory Panel on Research Ethics is the main body concerned with setting standards for ethical research practice in Canada, other organizations concerned with research ethics need to be considered and included in the conversation. The Canadian Association of Research Ethics Boards (CAREB) is a national organization representing REBs. A review of documentation and linked resources on the CAREB website provides very little guidance on IRE, except for one link in the resources section, from which researchers can access an "Internet Research Ethics" blog written by a masters student. So, little guidance on IRE appears to be available from the non-membership sections of the CAREB website.

The expertise of individuals on REBs, and their knowledge of IRE, is an issue. In 2009, Buchanan and Ess conducted a survey of ethics review boards at post-secondary institutions in the United States, known as Institutional Review Boards (IRBs), to gauge their knowledge and use of IRE. They found that $62 \%$ did not use guidelines to evaluate internet research and that while $60 \%$ required or recommended training on general ethics for their board members, only $9 \%$ required or recommended training on IRE. Internet research was identified by almost half of the participants as an area of importance and/ or concern. Even though this research was conducted in the US, a search of ethics information on major Canadian universities websites finds that very few include information on IRE, implying that a similar situation exists in Canada. CAREB is an organization well placed to demonstrate leadership and play a significant role in the development, promotion, and sharing of national guidelines and best practices for ethical internet research.

The Association for Educational Communications and Technology (AECT) is a professional organization of educators who are particularly interested in the role of technology in teaching and learning. An area of focus and current research for educational technologists is the potential of the internet to be an authentic learning environment, for example, reflective blogs, virtual games, and collaborative wikis (Januszewski \& Molenda, 2008). The AECT does not currently offer an established code of research ethics to guide the work by its members; however the professional code of ethics does highlight the need for educational technologist to stay informed of current and sound ethical research guidelines (Welliver, 2001). The association does point researchers to other professional organizations for guidance on research ethics and asserts in their own professional ethics statement that members "shall conduct research and practice using professionally accepted guides and procedures" (Welliver, 2001, p. 297). As educational technologists engage in studying teaching and learning in online settings, it is essential that they too be fully engaged in the conversation about IRE and the development of guidelines.

\section{Discussion}

Many ethical issues have been highlighted in this review of IRE and consideration must be given to accessibility of information, the nature of the content, informed consent, the perceived privacy of the site/group members, the need for anonymity, and the challenges of communicating through electronic means. We call for immediate action on the Canadian ethics policy gap to include review and consolidation of current research on IRE, additional research into the many questions and uncertainties that surround IRE, the creation of a new IRE working committee, and the revision of the current TCPC2 to include guidelines and cases for analysis of the practice of ethical research on the internet. 
One of the most significant IRE debates involves the question of private versus the public online world. Ongoing consultation with expert colleagues is an important and accepted part of making ethical decisions (McKee \& Porter, 2009). Additional Canadian research is needed to examine the practices of researchers, along with the preferences of users and members of various online communities regarding this divide. What proportion of people who post on the internet understand that their posts become public and archived information? How many people perceive or expect norms of confidentiality in the physical world to prevail in online spaces? Should there be generational, regional, and cultural differences in how ethical considerations are applied to online content and discourse? The work by McKee and Porter (2009), King (1996), and Estalella and Ardevol (2007) suggested that research participants may have strong feelings towards these issues and their research highlights the need for additional studies to provide insight into different participants' views on the private versus public issue, along with issues of recruitment, informed consent, and confidentiality.

Although it appears the United States is dealing with similar policy and practice gaps (Schrag, 2011), it would be worthwhile to examine how other countries, outside Canada and the United States, are dealing with issues of IRE. McKee and Porter (2009) suggested that some European nations have made advances regarding the development of IRE guidelines. Insights to inform the next version of Canada's TCPS may be gleaned from an international review of IRE research and practice.

It is important to recognize that ethical internet research may best be seen as "a process and a product of negotiation between the parties and not just a formal procedure" (Estalella \& Ardevol, 2007, p. 42). REBs will have to become more agile, interactive, and responsive to contextually, temporally, and situation-specific ethical concerns and methods brought forward by online researchers. While some guidelines are available to help inform researchers and suggest ethically sound research practices, there are still too many grey areas and emerging questions that may lead different REBs to overestimate or underestimate risk in applying very different levels of caution and constraint to online research across the country. Canada currently has no defined guidelines for IRE, which suggests that this is an area deserving greater attention by the scholarly community. McKee and Porter (2009) advocated for the value of heuristics, which are open-ended questions, prompts, or visual grids, to aid in the process of deliberation, consultation, and ethical review of internet research. Clearly, a current set of Canadian guidelines for internet research ethics is needed to make conversations about online research ethics more equitable between the REBs and researchers. Given the increasing prevalence of internet research, REBs and researchers cannot wait another 10 years for the current TCPS2 to be revised. We recommend that at least one case, similar to the ones published in McKee and Porter (2009), be added to the required TCPS2: CORE Tutorial to engage faculty and graduate researchers in questions and issues to do with IRE. Timelier attention to IRE is needed through the establishment of a new working committee to investigate the current and best practices of researchers and REBs regarding internet research, and to propose changes to the existing TCPS2 guidelines. It is important that key players are involved in these conversations, including the Tri-Council, the Canadian Association of Research Ethics Boards, and the Association for Educational Communications and Technology, and that these conversations are informed by the latest research and emerging practices of IRE. 
Good ethical practices for internet research are complex. Those in the research community who undertake the development of better policy must consider the situational, contextual, and temporal aspects of IRE in the development of flexible, open-ended, and responsive guidelines that address the complexity and diversity of online research spaces. Therefore, we are not suggesting the development of hard and fast decision rules, but the creation of systematic, collaborative, and multidisciplinary guidelines and frameworks (McKee \& Porter, 2009), complex IRE cases for education and analysis, and open-access sharing of best practices that will aid researchers and REBs in open and transparent decision making for the conduct of ethical internet research. These guidelines should include contextual examples and exemplars of internet research from around the globe.

\section{Conclusion}

Educational and social science researchers should continue to seek better and more responsive guidelines that describe how to deal with ethical issues arising in various online research situations, contexts, and conditions and to educate the community by publishing and sharing their practices, findings, and solutions. The Tri-Council needs to revise the current TCPS2 to specifically address IRE. REBs, responsible practitioners, researchers and scholars need to become informed of current ethical best practices and make every effort to use appropriate ethical guidelines when designing and conducting research using the internet. Further research investigating the complexities of ethical decision making in internet research and collaboration between professional associations that contribute to the development of a clear set of guidelines would aid greatly in this endeavour.

\section{Acknowledgements}

The authors thank Dr. Sylvie Roy, Faculty of Education, University of Calgary, for completing the English-to-French translation of the Abstract.

\section{References}

Bassett, E. H., \& O' Riordan, K. (2002). Ethics of Internet research: Contesting the human subjects research model. Ethics and Information, 4, 233-247.

Beaulieu, A., \& Estalella, A. (2011). Rethinking research ethics for mediated settings. Information, Communication \& Society, 15(1), 1-20.

Buchanan, E. A., \& Ess, C. M. (2009). Internet research ethics and institutional review board: Current practices and issues. SIGCAS Computers and Society, 39(3), 43-49.

Buchanan, E. A., \& Ess, C. M. (2008). Internet research ethics: The field and its critical issues. In K. E. Himma \& H. T. Tavani (Eds.), The handbook of information and computer ethics (273-292). New Jersey: John Wiley and Sons.

Canadian Intellectual Property Office. (2008). A guide to copyrights. Retrieved fromhttp://www.cipo.ic.gc.ca/eic/site/cipointernet-internetopic.nsf/vwapj/2010 guidedroitsdauteur-2010guidecopyrights-eng.pdf/\$file/2010guidedroitsdauteur2010guidecopyrights-eng.pdf. 
CIHR (Canadian Institute of Health Research), Natural Sciences and Engineering Research Council of Canada, and Social Sciences and Humanities Research Council of Canada. (2010a). Tri-Council policy statement: Ethical conduct for research involving humans. Retrieved from http://www.pre.ethics.gc.ca/eng/policy-politique/initiatives/ tcps2-eptc2/Default

CIHR (Canadian Institute of Health Research), \& Queen's University. (2010b). TCPS 2: Core tutorial. Retrieved from http://tcps2core.ca/welcome.

Charbonneau, L. (2005). Ethics boards harming survey research, says York professor. University Affairs, June 6. Retrieved from: http://www.universityaffairs.ca/ethicsboards-harming-survey-research-says-york-professor.aspx.

Elgesem, D. (2002). What is special about the ethical issues in online research? Ethics and Information Technology, 4, 195-203.

Ess, C., \& the Association of Internet Researchers ethics working committee. (2002). Ethical decision-making and Internet research: Recommendations from the AoIR ethics working committee. Retrieved from http://www.aoir.org/reports/ethics.pdf

Estalella, A., \& Ardevol, E. (2007). Ética de campo: hacia una ética situada para lainvestigación etnográfica de internet. Forum: Qualitative Social Research, 8(3). Retrieved from http://nbn-resolving.de/urn:nbn:de:0114-fqso70328.

Esyenbach, G., \& Till, J. E. (2001). Ethical issues in qualitative research on internet communities, BMJ, 323, 1103-1105.

Frankel, M. S., \& Siang, S. (1999). Ethical and legal aspects of human subject research on the internet. Washington, DC: American Association for the Advancement of Science. Retrieved from http://www.aaas.org/spp/dspp/sfrl/projects/intres/main.htm.

Jacobsen, D. M. (2010). Teaching in a participatory digital world. Education Canada, 5O(3), 13-17. Retrieved from http://www.cea-ace.ca/education-canada/article/teachingparticipatory-digital-world.

Jacobsen, D. M., and Friesen, S. (2011). Web exclusive: Hands on vs. hands up: Technology-enabled knowledge building in high school. Education Canada, 51(3). Retrieved from http://www.cea-ace.ca/education-canada/article/web-exclusive-handsvs-hands-technology-enabled-knowledge-building-high-sch.

Januszewski, A., \& Molenda, M. (Eds.). (2008). Educational technology: A definition with commentary. New York: Lawrence Erlbaum Associates.

King, S. (1996). Researching Internet communities: Proposed ethical guidelines for the reporting of results. The Information Society, 12(2), 119-129.

Kitchin, H. A. (2007). Research ethics and the Internet: Negotiating Canada's TriCouncil policy statement. Halifax and Winnipeg: Fernwood.

McKee, H. A., \& Porter, J. E. (2009). The ethics of Internet research: A rhetorical, case-based process. New York, NY: Peter Lang.

Moreno, M. A., Frost, N. C., \& Christakis, D. A. (2008). Research ethics in the MySpace era. Pediatrics, 121, 157-161. 
National Committee for Research Ethics in the Social Sciences and the Humanities. (2003). Research ethics guideline for Internet research. Retrieved from http://www. etikkom.no/en/In-English/Publications/Internet-research-/

Schrag, Z. M. (2011). The case against ethics review in the social sciences. Research Ethics, 7(4), 120-131. doi: 10.1177/174701611100700402.

SSHWC (Social Sciences and Humanities Research Ethics Special Working Committee). (2008). Extending the spectrum: The TCPS and ethical issues in Internetbased research. Ottawa, ON: Interagency Advisory Panel and Secretariat on Research Ethics. Retrieved from http://www.pre.ethics.gc.ca/policy-politique/initiatives/docs/ Internet_Research_-_February_2008_-_EN.pdf.

Thomas, D., \& Brown, J. Seely (2011). A new culture of learning: Cultivating the imagination for a world of constant change. CreateSpace.

Walther, J. B. (2002). Research ethics in Internet-enabled research: Human subjects issues and methodological myopia. Ethics and Information Technology, 4, 205-216.

Welliver P. W. (Ed.). (2001). A code of professional ethics: A guide in the field of educational communications and technology. Bloomington, IL: Association for Educational Communications and Technology. Retrieved from http://www.aect.org/ Internet/Publications/ethics.

\section{Contact Information}

Jacqueline G. Warrell

University of Calgary

2500 University Dr. NW

Calgary, Alberta, Canada

T2N $1 \mathrm{~N} 4$

jgwarrel@ucalgary.ca

Jacqueline Warrell is a Ph.D. student in workplace and adult learning in the Faculty of Education, University of Calgary. Her research interests include informal learning, communities of practice, mentoring, identity development, educational technology, online learning, and social media. Jacqueline's doctoral research examines the informal learning and professional identity development of graduate students in online learning communities. She is interested in issues surrounding conducting research on the Internet, including research ethics and online methodologies.

Michele Jacobsen is an associate professor in educational technology in the Faculty of Education, University of Calgary. She studies technology-enabled learning and teaching in both real time and online kindergarten to Grade 12 classrooms and post-secondary education using case study and design-based research methodologies. Informed by current research from the learning sciences, Michele examines designs that shift instruction from standardized delivery and testing to participatory pedagogies in learning environments that sponsor knowledge building, intellectual engagement and assessment for learning. Michele interested in research ethics, especially the ongoing development of policy and guidelines to inform Internet research methodology. 